\title{
CONF- $950848--6$
}

Workshop on Diagnostics for ITER, Varenna. Italy, August 28-September 1. 1995

\section{APPLICATION OF INTERFEROMETRY AND FARADAY ROTATION TECHNIQUES FOR DENSITY MEASUREMENTS ON ITER}

\author{
R.T. Snider, 'T.N. Carlstrom, ${ }^{1}$ C.H. Ma ${ }^{2}$ W.A. Peebles ${ }^{3}$ \\ 1 General Atomics, P.O. Box 85608, San Diego, CA 92186-9784 \\ 2 Oak Ridge National Laboratory, P.O. Box 2009, Oak Ridge, TN 37831 \\ ${ }^{3}$ University of California at Los Angeles, CA $90024-1597$
}

TH:

\begin{abstract}
There is a need for real time, reliable density measurement for density control, compatible with the restricted access and radiation environment on ITER. Line average density measurements using microwave or laser interferometry techniques have proven to be robust and reliable for density control on contemporary tokamaks. In ITER, the large path length, high density and density gradients, limit the wavelength of a probing beam to shorter then about $50 \mu \mathrm{m}$ due to refraction effects. In this paper we consider the design of short wavelength vibration compensated interferometers and Faraday rotation techniques for density measurements on ITER. These techniques allow operation of the diagnostics without a prohibitively large vibration isolated structure and permits the optics to be mounted directly on the radial port plugs on ITER. A beam path designed for $10.6 \mu \mathrm{m}$ (CO2 laser) with a tangential path through the plasma allows both an interferometer and a Faraday rotation measurement of the line average density with good density resolution while avoiding refraction problems. Plasma effects on the probing beams and design tradeoffs will be discussed along with radiation and long pulse issues. A proposed layout of the diagnostic for ITER will be present.
\end{abstract}

\section{INTRODUCTION}

The measurement of the electron density in large tokamaks play a critical role in the operation and understanding of the plasmas produced. Active feedback control of the density is routinely used in present day tokamaks to optimize the performance of the plasma, to avoid density limit disruptions and to perform systematic studies with the density as an independent variable. In the next generation of machines, such as the Intemational Thermonuclear Experimental Reactor (ITER), density measurements and active density control will take on an even more important role due to the increased seriousness of disruptions and the added element of bum control. Many of the present critical areas of concerns for the ITER design team, such as fueling and disruption avoidance assume very accarate and reliable density measurements. 1

Line average density measurements using microwave or laser interferometry techniques have proven to be robust and reliable for density control in tokamaks and can provide the accuracy needed for ITER. Another density measurement technique proposed by Jobes ${ }^{2}$ determines the density by measuring the Faraday rotation of a probing beam tangent to the toroidal field. While the Faraday rotation method has yet to be demonstrated on a tokamak it has some advantages over interferometer techniques particularly in long pulse operation since it does not rely on keeping track of fringe shifts. A density interferometer and a Faraday rotation density measurement can both use the same optics path and port interface and can probably use the same probing beam allowing both measurements to be made simultaneously. There are distinct advantages in using both techniques in terms of complimentary information and improved reliability. 
ITER presents significantly new and difficull challenges to interferometer and Faraday rotation density measurements due to the large plasms path length, long puise length, high electron densiry, severely restricted access and the nuclear environment. A beam path designed for $10.6 \mu \mathrm{m}\left(\mathrm{CO}_{2}\right.$ laser) with a path tangential to the toroidal field at the mid plane through the plasma allows both a vibration compensated interferometer and a Faraday rotation measurement that address these ITER relevant issues without penetrntions or disturbance of the ITER shield modules other then the radial port plugs. This design has minimal impact on the ITER machine design and uses proven techniques that are well understood and pose litule technical risk.

\section{FARADAY ROTATION AND INTERFEROMETRY TECHROUES FOR ELECTRON DENSITY MEASUREMENTS}

Laser interferometry is a well established method for measuring electron density in high temperature plasmas going back to the first demonstration by Ashby and Jephcott in $1965 .^{3}$ In a high temperature plasma the change in the refractive index is dominated by the electrons and, in a tokamak plasma can cause significant displacement deflection and phase lag of a probing beam. The phase lag caused by the electron density $\left(n_{e}\right)$ is given approximately by $\phi=\left(\pi \lambda_{n_{c}}\right.$ Ind $\ell$ where $n_{c}$ is the cutoff frequency, $\left[\left(n_{c}=4 \pi c^{2} \varepsilon_{0} m_{d} / e^{2}\right)\right] e$ is the electron change, $c$ is the speed of light in vacuum, $\varepsilon_{0}$ is the dielectric constant, me is the electron mass. Many techniques have been developed to measure this phase lag but the most common technique now used on tokamaks is to split the laser beam into a plasma probing beam and a reference beam with the reference beam Doppler shifted, either by moving mirrors, 5 moving gratings ${ }^{6}$ or acoustic optic cells?. The beams are combined after the plasmn probing beam passes through the plasma. The resulting mixed beam has a beat frequency approximately equal to the Doppler frequency shift. due to interference between the plasma probing beam and the Doppler shifted reference beam. The plasma induced phase shift is then measured by measuring the difference between the period of the beat frequency (by measuring the time between zero crossings) and the period of the Doppler shift. The change in the line average density during each cycle of the beat period is the quantity measured and a running total of these phase shifts gives the total line average density. Any loss of this running total, or integral of phase shifts during a plasma discharge results in the loss of the density measurement.

Mechanical motion of the mirrors in the direction of beam propagation produce phase shifts $(\Delta \phi=2 \pi \Delta(\lambda)$ in the plasma probing beam. Vibration isolation stuctures are impractical on a machine the size of ITER, particularly with the restricted port access. Further, at the short wavelengths appropriate for IIER even a vibracion insulated structure cannot reduce vibrations to an acceptable level. However, second wavelength interferometer can be used to compensate for any motion of the optical components. This allows the optical components, such as the retroreflectors and relay mirrors, to be directly mounted onto the vacuum vessel or port structares.

A measurement of the Faraday rotation of a probing beam that is tangential to the toroidal field at the midplane can also be used to monitor the plasma density. This technique was proposed for ITER by Jobes ${ }^{2}$ and has several advantages over interferometry. The measurement does not depend on the past history of the plasma discharge (the absolute measurement of the Faraday rotation is all that is required as long as the total Faraday rotation is less than $2 \pi \pi$ ) and vibrarion of the optics do not effect the measurement. A linearly polarized beam propagating in the direction of the magnetic field experiences a rotation in the polarizarion of $\Omega=\left[\lambda^{3} e^{3} /\left(8 \pi^{2}\right.\right.$ $\left.\left.\left.c^{3} \varepsilon_{0} m_{e}^{2}\right)\right]_{n} B_{\| l} d \ell.\right]$ where $B_{\|}$is the magnetic field parallel to the beam propagarion. At the midplane the magnetic field is just the toroidal field $\left(B_{T}=B_{0} R_{0} / R\right.$ where $B_{T}$ is the toroidal field, $B_{0}$ is the toroidal field at the center of the discharge, $R_{0}$ is the major radius at the center of the discharge and $R$ is the radial distance from the centerline of the tokamak). If the beam is off midplane, as would almost certainly be the case in ITER, the Poloidal field would have some component along the beam line and would have some effect on the polarization. However the effect is small and antisymetric and should not canse a significant error. An Abel inversion is required in onder to extract the density from the dam (i.e. a single channel does not give the line average density as with an interferometer), however as discussed earlier because of the toroidal symmetry a rough Abel inversion can be made with few channels. Further it is likely that the densiry control function can be done with a single channel $\int_{n_{e}} B d \ell$. measurement just as the line average density is used in existing tokamaks for density control and not a density profile or the peak density.

The tangential geometry proposed for a density measurement on ITER allows the possibility of making both an interferometer measurement and the Faraday rotation measurement using the same optics and indeed the same laser beam. Further the measurements are complementary in that the interferometer for a given wavelength has better density resolution given the current demonstrated detector technology, while the Faraday rotation does not have the 


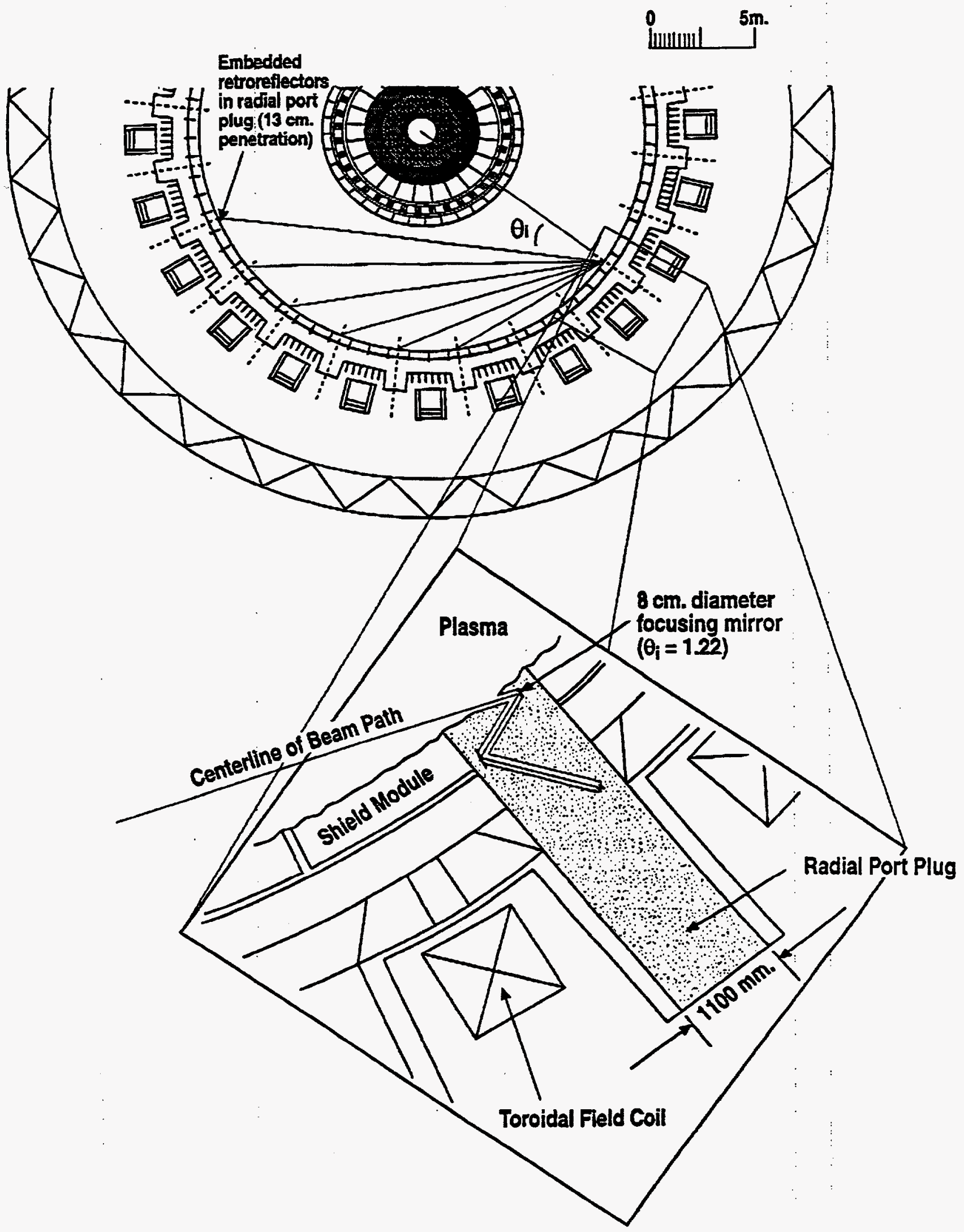


long pulse problem of keeping track of fringe shifts. Also the two methods give slightly different information that could be used to improve the density profile information from a given set of beamlines. -

\section{DIAGNOSTIC GEOMETRY}

The port access on ITER will be severely limited and will limit the lines of sight available for probing beams. As many as six horizontal lines of sight at the midplane, tangential to the toroidal field, can be realized in ITER using the existing radial ports without modifying the shield/blanket modules or any other machine structure other than the radial port plugs as shown in Fig. 1. Penetrations or modifications to the shield/blanket modules are highly undesirable and costly due to the complexity and the remote handling requirements of the modules. Further, peneurations through the shield/blanket modules (either inboard or outboard) compromise the nuclear shielding of the super conducting coils. These considerations restrict or perhaps eliminate geometry's other then those similar to the proposed layout, such as radial lines of sight with retroreflectors mounted in the inboard shield or vertical lines of sight with retroreflectors mounted in the lower part of the outboard shield/blanket module in line with the vertical port.

The proposed tangential layout has other advantages over either radial or vertical sight lines: 1.) The symmetry about the center post allows a straight forward Abel inversion of the line integrated data yielding rough density profiles from relatively few channels. While the six sight lines proposed could not give fine, detailed density profiles, the profiles produced would be adequate for density profile: control measurements and for most physics analysis, such as confinement time studies. 2.) The tangential sight lines always sample the entire profile (in particular the center) regardless of the Shafranov shift. 3.) The tangential geometry allows the option of using the Faraday rotation of the probing beam as a density monitor.

Figure 1. The proposed layout uses a single radial port for the entrance and exit beamlines for all of the sight lines and retroreflectors embedded in other radial port plugs. Relay optics in the radiai port transport the beams through a shiclding labyrinth and are then fanned out across the plasma and returned by the embedded retroreflectors.

\section{CONSTRAINTS ON WAVELENGTHS}

In considering possible wavelengths for a probing beam for a plasma density measuremient (either an interferometer or a Faraday rotation measurement) several effects must be taken into account. 4 The refraction and diffraction of the probing beam favor short wavelengths while 
density resolution, optical surface quality of plasma facing components and in the case of interferometry, mechanical vibrations favor longer wavelengths. Tradeoffs between these competing affects drive the usable gavelengths for ITER into a very narrow range, between roughly $1 \mathrm{\mu m}$ and $50 \mu \mathrm{m}$.

The variation of the angle of deviation $\left(\alpha_{\mathrm{m}}\right)$ of a $119 \mu \mathrm{m}$ beam as a function of tangential angle of incidence $\left(\theta_{i}\right.$ defined in Fig- 1$)$ due to refraction, is shown in Fig. 2 for an ITER-sized plasma with a central electron density of $1.1 \times 10^{20} \mathrm{~m}^{-3}$ for four density profile shapes. ${ }^{8}$ Also shown in Fig. 2 is the angular locations of the proposed tangential lines of sight shown in Fig. 1. The refractive bending of the probing beam results in a translation of the beam at the location of the retum retroreflector (di) which is approximately proportional to no 22 for small deviacions and is plotted in Fig. 3 as a function of wavelength for a family of tangential angles of incidence for a parabolic density profile. A beam with wavelength of $10.6 \mu \mathrm{m}$ has a maximum deflection of less then $1 \mathrm{~mm}$ and is an order of magnitude smaller then typical beam diameters and can. be ignored. At $50 \mu \mathrm{m}$ the deflection is about $1 \mathrm{~cm}$ and at $100 \mu \mathrm{m}$ the deflection is several centimeters. The large displacements caused by refraction above $50 \mu \mathrm{m}$ make alignment of the beam line difficult (or at least adds significantly to the beam alignment problem).

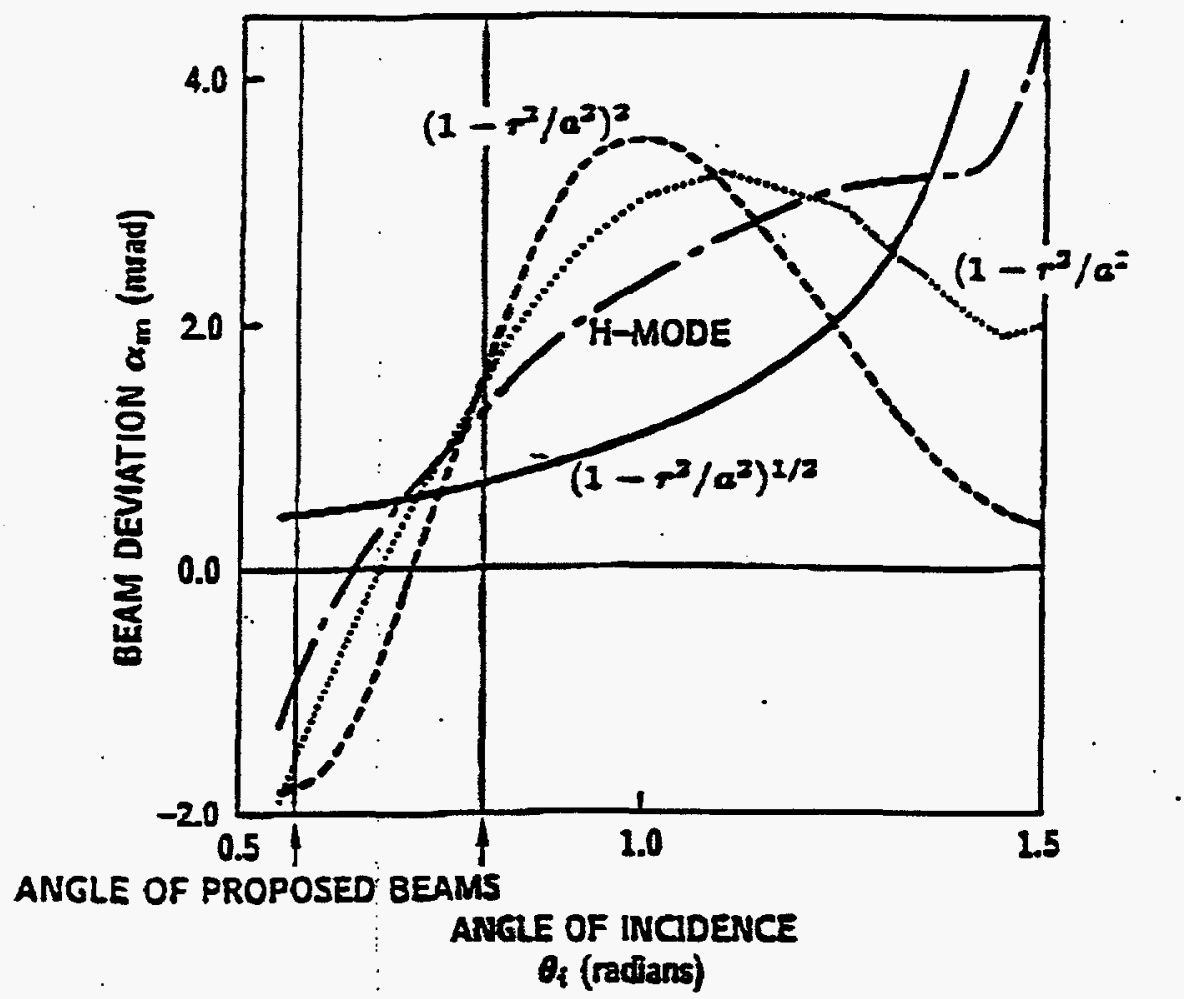

Figure 2. The variation of the angle of deviation $\left(\alpha_{m}\right)$ of a $119 \mu \mathrm{m}$ beam as a function of the tangential angle of incidence $\left(\theta_{i}\right)$ for an TTER-sized plasma with a centrai electron density of $1.1 \times 10^{20} \mathrm{~m}$. The characteristic $\mathrm{H}$-mode density profile has an edge density gradient of $3.21 \times 10^{21} \mathrm{~m}^{-4}$ with constant density across the central region.

The divergence and the diameter of the probing beam and the path length determine the dimensions of the plasma facing optics and the opening in the port plugs and thus play a large role in determining the bounds (on the long wavelength end) on the wavelength. The path length between the retroreflector and the rurning mirror $\left(\mathrm{Z}_{0}\right)$ shown in Fig. 2 is about $20 \mathrm{~m}$. We want to minimize the size of the retroreflector in order to cause the smallest perturbation in the part plugs that house the retroreflectors. For a Gaussian beam the laser spot size that contains $95 \%$ of the power at the beam waist (set to be at the retroreflector) is $d_{0}=2 \pi\left(2 \lambda Z_{0} / \pi\right)^{1 / 2}$ where $\eta$ is a factor representing the increase in the divergence of the laser beam above the defraction limit (commercially available $\mathrm{CO}_{2}$ lasers are available with $\pi=2$ for example). The turning mirror shown in Pig. 1, then would have to be a focusing mirror and the laser spot at the turning mirror would be $\mathrm{d}_{0}=\sqrt{2} \mathrm{~d}_{0}$.

With all of these factors included $d_{0}=4 \eta\left(2 \lambda Z_{0} / \pi\right)^{1 / 2}$ for $10.6 \mu \mathrm{m}$, the retroreflector would be at least $9.2 \mathrm{~cm}$ (assuming $\eta=2$ ) and the turning mirror would be $13 \mathrm{~cm}$ (for $\theta .=0.77$ ), as can be seen in Fig. I the path length changes as a function of $\theta$ : and thus the optics sizes change somewhat for the various paths. 


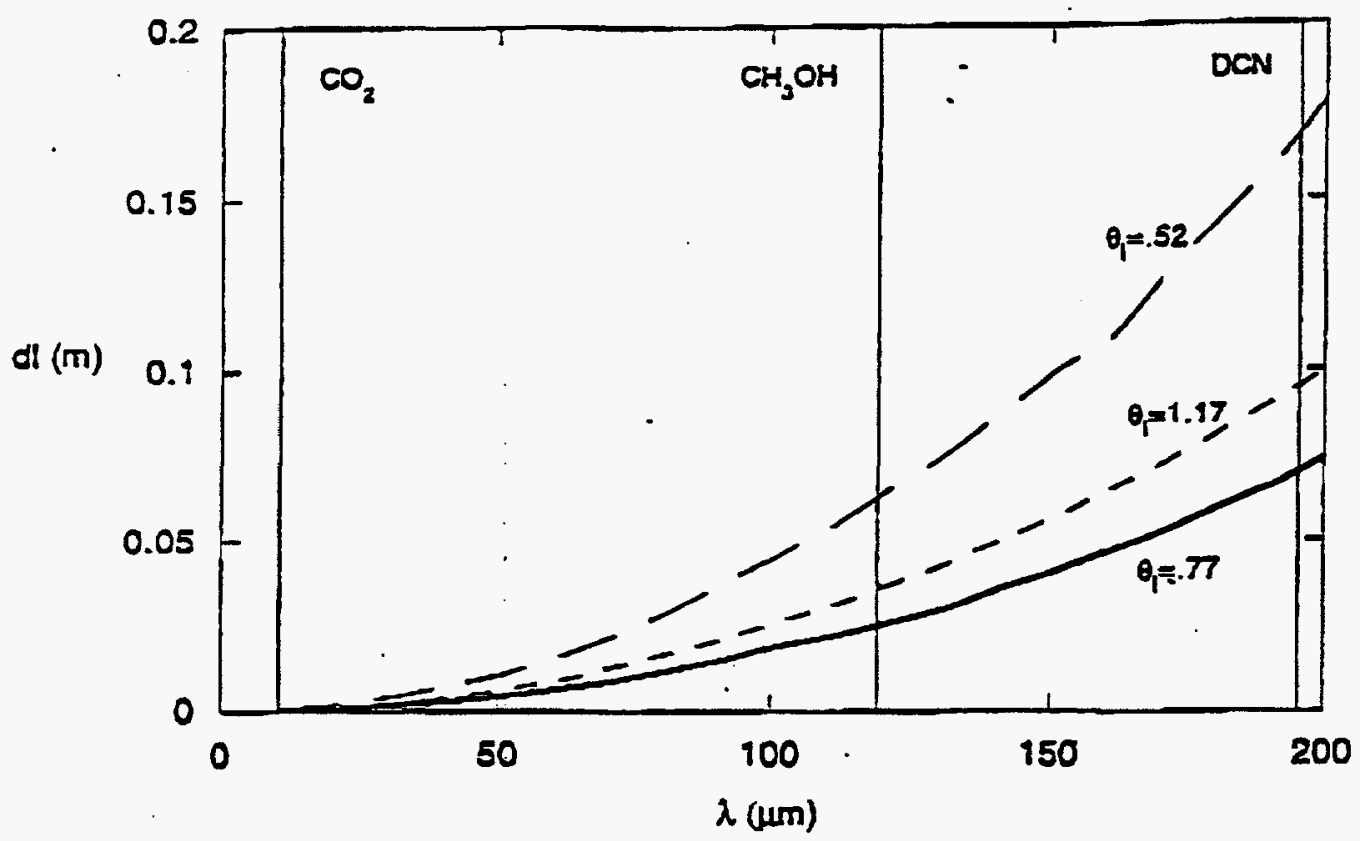

Figure 3. The transiation of a beam due to refraction at the location of the renum retroreflector as a function of wavelength.

The density resolution for an interferometer is determined by the total phase shift caused by the plasma and the detector/electronics resolution of the phase shift while the density resolution for a Faraday rotation density measurement is likewise determined by the Faraday rotation caused by the plasma and the detector/electronics resolution of that polarization shift. Table I summarizes the plasma effects for various wavelengths including the total number of fringe shifts and the Faraday rotation. The last two columns in Table 1. list the density resolution for both types of measurements assuming detector/electronics with $1 / 100$ of a fringe resolution for the interferometer and $1 / 1000$ of a radian resolution of the polarization for the Faraday rotation measurement. These numbers are consistent with demonstrated detector/electronics, so of course these are not firm numbers and improvements are probable. It should be noted that the density resolution for a vibration compensated interferometer is reduced because of the use of the second wavelength interferometer and that the density resolution decreases as the difference berween the wavelengths decreases as shown in Fig. 4. With existing technology a $10.6 \mu \mathrm{m}$ interferometer would give line average density resolution of better than $0.2 \%$, while the Faraday rotation measurement would give $\mathrm{a} \overline{\mathrm{nB}}$ resolution of about $5 \%$ at low densities.

Table 1. Summary of plasma effects for various wavelengths for a tangantial $(\theta:=0.77)$ double pass in IIER. The density resolutions assume $\Delta \phi$ resolution of 11100 radian and polarization resolution of 1/1000 radian.

$\theta_{i}$

\begin{tabular}{|c|c|c|c|c|c|c|c|}
\hline$\lambda(\mu m)$ & $\begin{array}{c}\text { Port } \\
\text { Penetration } \\
\text { Size }(\mathrm{cm})\end{array}$ & $\begin{array}{l}\theta:=0.77 \\
\mathrm{n}_{\mathrm{f}}(0) \\
\times 10^{9} \mathrm{~m}^{-3}\end{array}$ & $\begin{array}{l}\text { Toeal } \\
\text { Phase } \\
\text { Shift } \\
\text { (rad) }\end{array}$ & $\begin{array}{l}\text { Equivalent } \\
\text { Path } \\
\text { Length }\end{array}$ & $\begin{array}{l}\text { Faraday } \\
\text { Rotation } \\
\text { (rad) }\end{array}$ & $\begin{array}{c}\text { Interferometer } \\
\Delta \bar{n}_{e} / \bar{n} \\
\left(\times 10^{2}\right)\end{array}$ & $\begin{array}{l}\text { Faraday } \\
\text { Rotation } \\
\Delta \bar{n}_{e} B / \bar{n}_{e} B \\
\left(\times 10^{-2}\right)\end{array}$ \\
\hline 195 & $74 / 91$ & 13 & 1969 & $61 \mathrm{~mm}$ & 73 & $5 \times 10^{-4}$ & $1.4 \times 10^{-3}$ \\
\hline & & 1 & 151 & $4.6 \mathrm{~mm}$ & 5.6 & $6.6 \times 10^{-3}$ & 1.8 \\
\hline 119 & $44 / 57$ & 13. & 1202 & $23 \mathrm{~mm}$ & 27 & $8 \times 10^{-3}$ & $3.7 \times 10^{-3}$ \\
\hline & & 1. & 92 & $1.7 \mathrm{~mm}$ & 2.1 & $10^{-2}$ & $4.8 \times 10^{-2}$ \\
\hline 50 & $22 / 30$ & 13 & 505 & $4 \mathrm{~mm}$ & 4.8 & $2 \times 10^{-3}$ & $2 \times 10^{-2}$ \\
\hline & & $i$ & 39 & $300 \mu \mathrm{m}$ & 0.37 & $2.6 \times 10^{-2}$ & $2.7 \times 10^{-2}$ \\
\hline 10.6 & $10 / 13$ & 13 & 107 & $180 \mu \mathrm{m}$ & 0.21 & $9.3 \times 10^{-3}$ & 0.48 \\
\hline & & 1 & 8 & $14 \mathrm{\mu m}$ & $1.6 \times 10^{-2}$ & 0.12 & 6.2 \\
\hline 3.39 & $6 / 8$ & 13 & 34 & $18 \mu \mathrm{m}$ & $2.2 \times 10^{-2}$ & $2.9 \times 10^{-2}$ & 4.5 \\
\hline & & 1 & 2.6 & $1.4 \mu \mathrm{m}$ & $1.7 \times 10^{-3}$ & 0.38 & 60 \\
\hline 1 & $3 / 4$ & 13 & 10 & $1.6 \mu \mathrm{m}$ & $1.9 \times 10^{-3}$ & 0.1 & 52 \\
\hline & & 1 & 0.78 & $0.1 \mathrm{\mu m}$ & $1.5 \times 10^{-4}$ & 1.3 & 666 \\
\hline
\end{tabular}


All of the optical surfaces will require flatness of $/ 4$ across the diameter of the component and surface roughness to be less then about $\lambda 10$ for the life of ITER. Metal front surface mirrors should not experience degradation due to neutron damage however the plasma facing optical surfaces will be subjected to particle bombardment from the plasma. The particle flux can cause sputter damage and coating of the surface. While it is not clear at this time how badly the optical surfaces will be degraded in ITER from the particle flux it is advantageous to use as long a wavelength as possible to minimize the effect on the density measurement. From existing tokamak experience, primarily the shor wavelength interferometer used on DUI-D, visible light is problemaric due to coating from the plasma on optical components close to the plasma. While the coating and damage that optical components will experience on ITER will be different (different wall material, radiation fluxes and fluence and energy distribution) it is likely that the optical surface degradation due to coating and damage will be worse on IIER then on existing tokamaks. This then argues for long wavelengths, at least longer then visible light.

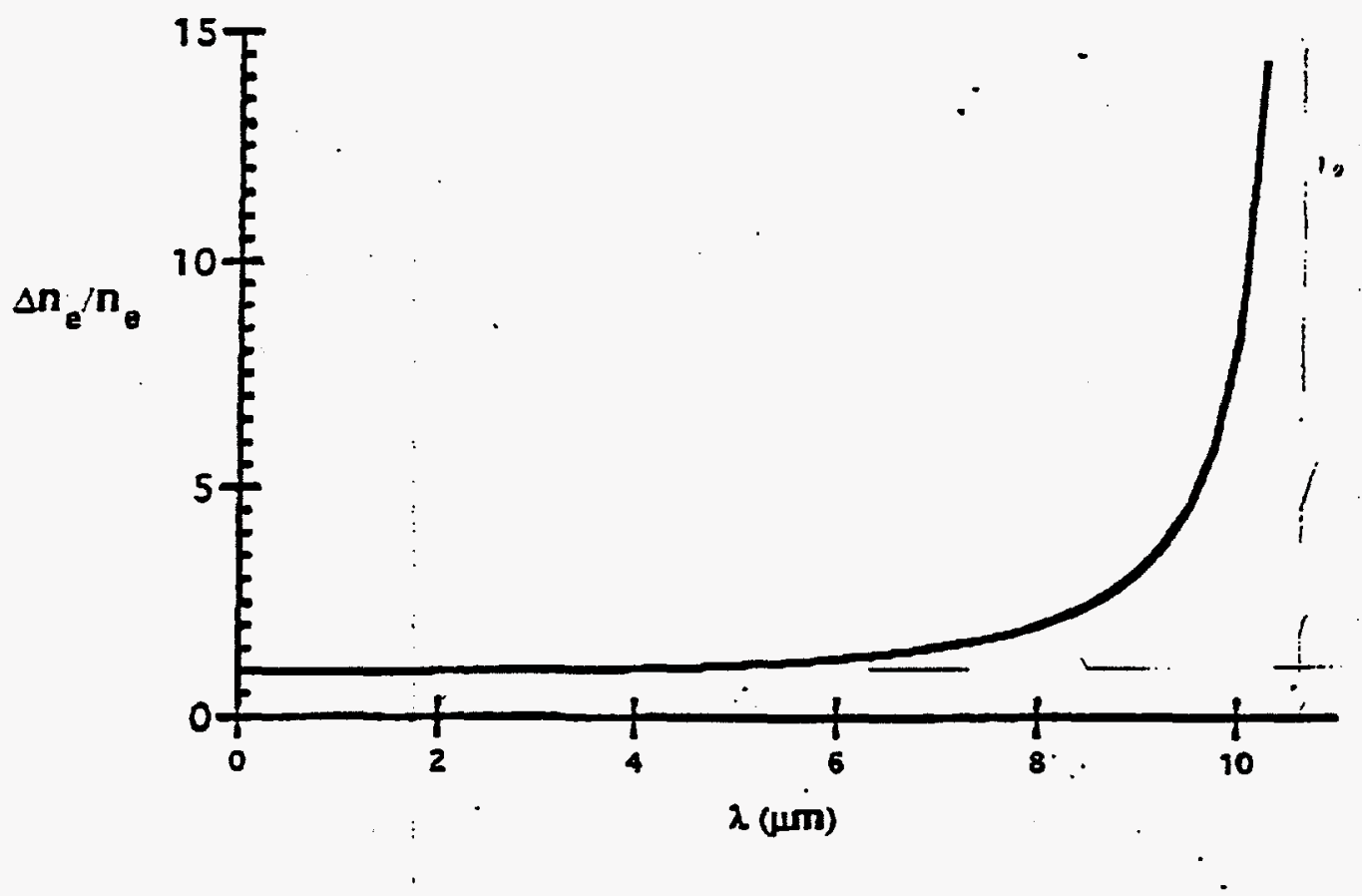

Figure 4. Reduction of the density resolution of a two color interferometer over a single color interferometer as a function of the second color wavelength for a $\mathrm{CO}_{2}(10.6)$ primary interferometer.

The constraints on the wavelength of a probing beam discussed above place practical limits on the wavelengths of between roughly $1 \mu \mathrm{m}$ and $50 \mathrm{\mu m}$ with intermediate wavelengths preferred. With this in mind we propose a $10.6 \mu \mathrm{m}\left(\mathrm{CO}_{2}\right)$ system for ITER including both a Faraday rotation measurement and a vibration compensated interferometer using $3: 39 \mathrm{\mu m}$ (HeNe) as the second wavelength. Commercially available lasers with adequate power are available, detectors and window material are well developed and a interferometer using these wavelengths has been operational on the DIII-D tokamak for several years. 10 Fig. 5 shows reprocative time traces for a DIII-D discharge including the line average density and the vibration of the vacuum vessel measurement by the two color interferometer. The penetrations through the port shield plugs are manageable $(13 \mathrm{~cm})$, the density resolution for both the Faraday rotation measurement and the interferometer are much better then required by ITER. Overall the proposed $10.6 \mathrm{~mm}$ and $3.39 \mu \mathrm{m}$ system represents a minimum of technical risk and can easily meet the specifications required by ITER.

\section{RADIATION}

The plasma facing optical components will experience neutron fluxes and fluences that will approach the first wall leveis. The recessed, tangential geometry shown in Fig. 1 allows some shielding of the retroreflectors and the finial turning mirrors, but the neutron fluence experienced by those components will be close to $5 \times 10^{17}$ neutrons $/ \mathrm{cm}^{2}$. Radiation testing of optical 

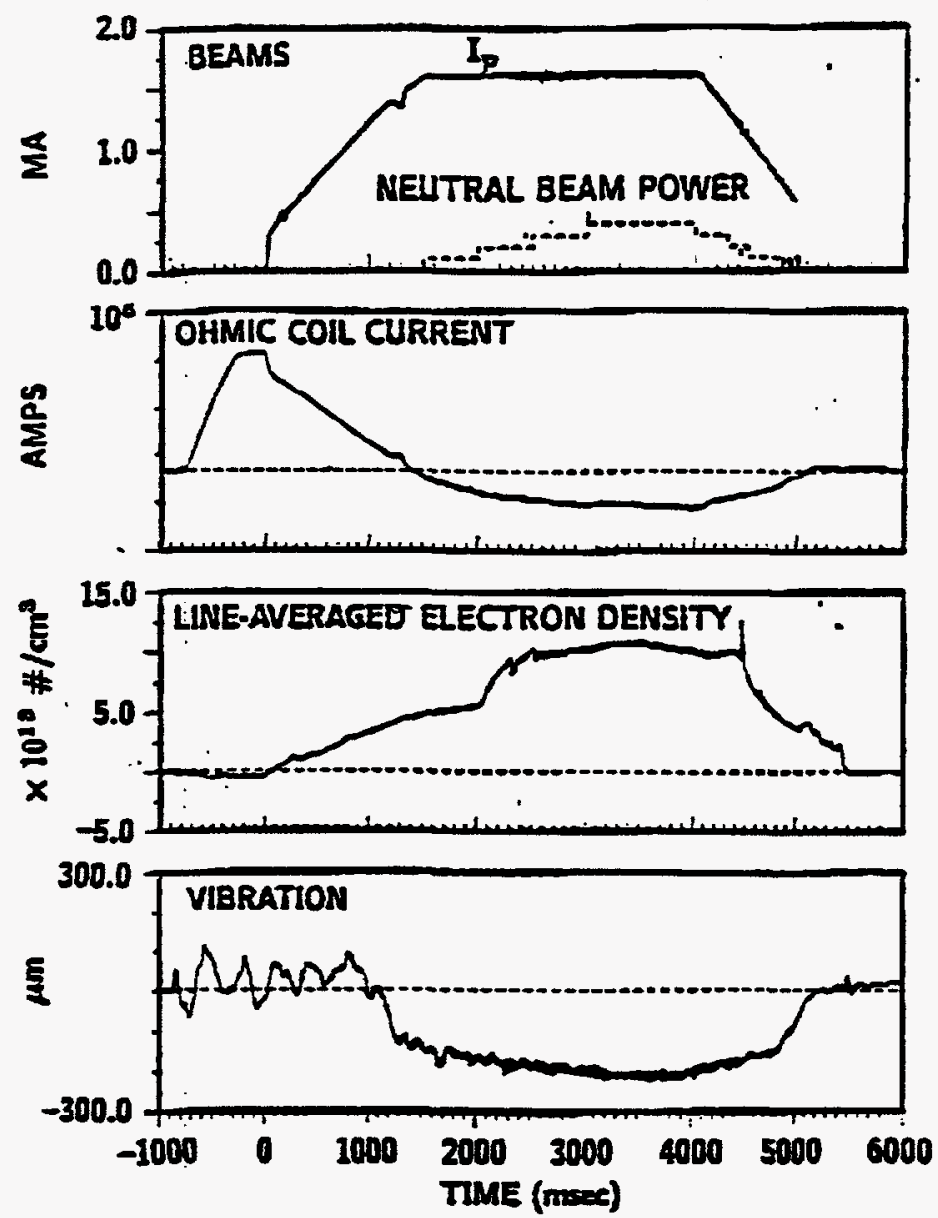

Figure 5. A viabration compensated inverferomerer using lasers at 10.6 and $3.39 \mathrm{~mm}$ and a retroreflector mounted directly on the vacuum vessel has been build and operated on the DIII-D tokamak.

components indicate that first surface, metal mirrors will survive these fluence levels. However coated mirrors and refractive optics almost certainly will not survive (very little radiation testing of Infra-red (IR) optic elements have been done at the ITER neutron levels, but the radiation testing of visible refractive optics indicate serious problems with radiation darkening and other damage 9 . For an interferometer or Faraday rotarion measurement darkening is less of a problem then intensity sensitive diagnostics since only the phase or polarization information is used, not the absolute intensity). This means that the first several optical elements closest to the plasma will have to be metal mirrors that relay the beams through a shielding labyrinth (shown in Fig. 1), to a place where the neutron levels are low enough for refractive optics to survive. This implies that the focus of radiation testing of IR refractive optics should be to determine the neutron flux and fluence that the element can survive which then defines how close to the plasma in the beam path the element can be placed.

\section{INTEGRATION OF DIAGNOSTIC DESIGNS}

The limited access and high machine interface costs of ITER makes it imperative that the diagnostic suite as a whole be optimized and that any synergistic advantages that exist among diagnostic systems be exploited. An example of such a complimentary set of diagnostics is the combination of reflectometry, coroidal interferometry and Faraday rotation measurement. The strengths and weaknesses of the systems are complimentary. The interferometry and Faraday rotation measurements provide reliable accurate core measurements in real time that can be simply Abel inverted, the addition of data from a reflectometer would further increase the accuracy of an inversion particulariy by defining the edge density. Determining edge density, parricularly the scrape-off density, is one of the biggest sources of error for a density inversion of line integrated density, while there are difficulties in making a central density measurement using reflectometry alone in ITER. Integrating the data from these systems to produce a composite profile is straightforward, such that together, a much more accurate, reliable and spatially 
detailed profile can be produced, with guaranteed core and edge measurements with good resolution. Combining the diagnostics as a single system reduces the rechnical risk of each and reduces the total ITER penetrations required and hence the total cost. To fully exploit this synergism requires that the concept of using the diagnostics together be considered during the design of these systems, not just combining the data after the systems are built.

\section{CONCLUSIONS}

The need for a reliable density measurement for piasma control on ITER within the considerable limitations imposed by the ITER environment has lead us to propose a limited array (six spatial channels or less shown in Fig. 1) of probing beams, tangential to the toroidal field, at the midplane of ITER employing wavelengths of $10.6 \mu \mathrm{m}$ and $3.39 \mu \mathrm{m}$. Both an interferometer and a Faraday rotation density measurement can be made at these wavelengths using the same beam path and even the same laser. The choice of tangential geometry and in particular the layout shown in Fig. 1 has minimal impact on the ITER device and requires no modification to the critical shield/blanket modules. Due to the large physical size and the harsh environment of ITER the choice of wavelength is severely limited by refractive and diffraction effects and by optical surface quality/survivability of the plasma facing components. Practical limits on the wavelengths are between roughly $1 \mu \mathrm{m}$ and $50 \mu \mathrm{m}$. The choice of $10.6 \mu \mathrm{m}$ and $3.39 \mu \mathrm{m}$ is driven by laser and detector availability. The proposed system is straightforward (at least as straightforward as is possible on ITER), has minimal impact on the IIER design, has a proven tokamak track record of reliability and can provide the density measurements needed for control of ITER plasmas.

The combination of an interferometer and a Faraday rotation measurement using the same diagnostic probing beam has synergistic advantages since the measurements are complimentary and provide different information that can be exploited to improve any density profiles. The integration of diagnostic designs to exploit other synergism's (such as reflectometry and interferometry) between diagnostic should be aggressively explored due to the restricted access and high cost of machine interfaces inherent in ITER.

\section{REFERENCES}

1. ITER Report TAC-95-15; TAC-JCT informal technical reviews.

2. F.C. Jobes and D.K. Mansfield, Rev. Sci. Instrum. 63, 5154 (1992).

3 Ashby, D.E. T.F. and Jephcott, D.F. Appl. Phys. Lett., 3, 13, (1963)

4. D. Viron, in Infrared and Millimeter Wave, edited by KJ. Button, (Academic, New York, 1979), Vol. 2.

5. D.R. Baker and S.-T. Lee, Rev. Sci. Instrum. 49, 919 (1978).

6. G. Dodel and W. Kunz, Infrared Physics 18, 773 (1978)

7. T.N. Carlstrom, D.R. Ahigren, and J. Croshie, Rev. Sci. Instrum. 59, 1063 (1988).

8. P. Gohil, et al., Phys. Rev. Lett 61, 1603 (1988).

9. E.H. Famum el al., J. Nucl. Mater. 219 (1995) 224.

10. R.T. Snider and T.N. Caristrom, Rev. Sci. Instrum., 63, 4979 (1992).

11. E.S. Doyle, TI. Rhodes, J.L. Doane and W.A. Peebles, Rev. Sci. Instrum. 66, 1233 (1995).

\section{DISCLAIMER}

\footnotetext{
This report was prepared as an account of work sponsored by an agency of the United States Government. Neither the United States Government nor any agency thereof, nor any of their employees, makes any warranty, express or implied, or assumes any legal liability or responsibility for the accuracy, completeness, or usefulness of any information, apparatus, product, or process disclosed, or represents that its use would not infringe privately owned rights. Reference herein to any specific commercial product, process, or service by trade name, trademark, manufacturer, or otherwise does not necessarily constitute or imply its endorsement, recommendation, or favoring by the United States Government or any agency thereof. The views and opinions of authors expressed herein do not necessarily state or reflect those of the United States Government or any agency thereof.
} 